This item was submitted to Loughborough's Research Repository by the author.

Items in Figshare are protected by copyright, with all rights reserved, unless otherwise indicated.

\title{
An unstructured-mesh atmospheric model for nonhydrostatic dynamics
}

PLEASE CITE THE PUBLISHED VERSION

http://dx.doi.org/10.1016/j.jcp.2013.07.027

PUBLISHER

(C) Elsevier

VERSION

AM (Accepted Manuscript)

LICENCE

CC BY-NC-ND 4.0

REPOSITORY RECORD

Smolarkiewicz, Piotr K., Joanna Szmelter, and Andrzej A. Wyszogrodzki. 2019. "An Unstructured-mesh Atmospheric Model for Nonhydrostatic Dynamics". figshare. https://hdl.handle.net/2134/13371. 
This item was submitted to Loughborough's Institutional Repository (https://dspace.lboro.ac.uk/) by the author and is made available under the following Creative Commons Licence conditions.

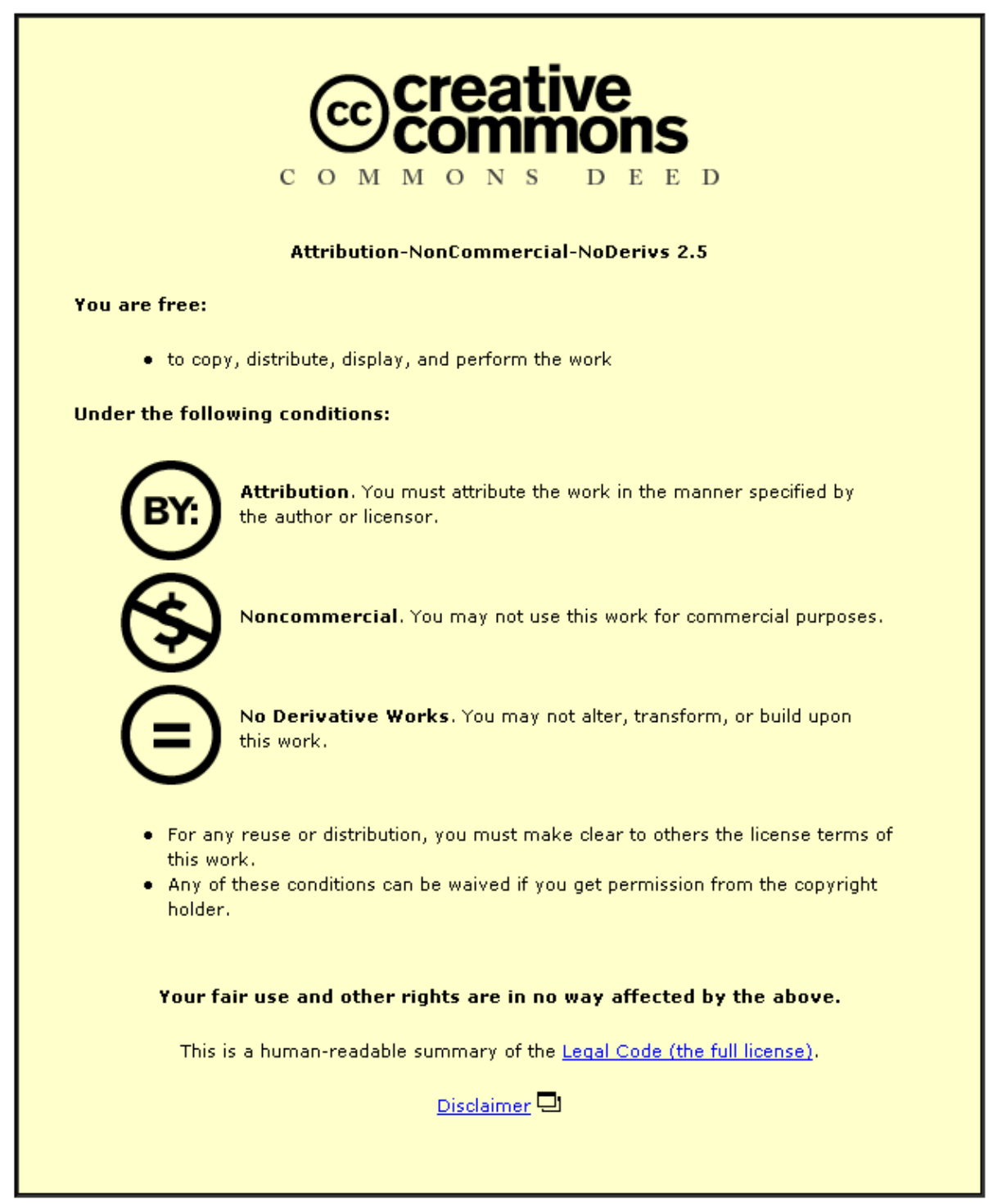

For the full text of this licence, please go to: http://creativecommons.org/licenses/by-nc-nd/2.5/ 


\title{
An unstructured-mesh atmospheric model for nonhydrostatic dynamics
}

\author{
Piotr K. Smolarkiewicz ${ }^{a}$, Joanna Szmelter ${ }^{\mathrm{b}}$, and \\ Andrzej A. Wyszogrodzki ${ }^{\mathrm{c}}$ \\ aEuropean Centre For Medium Range Weather Forecasts, Reading, RG2 9AX, UK \\ bLoughborough University, Leicestershire LE11 3TU, UK \\ ${ }^{\mathrm{c} N a t i o n a l}$ Center for Atmospheric Research, Boulder, C080307, USA
}

\begin{abstract}
A three-dimensional semi-implicit edge-based unstructured-mesh model is developed that integrates nonhydrostatic anelastic equations, suitable for simulation of small-to-mesoscale atmospheric flows. The model builds on nonoscillatory forwardin-time MPDATA approach using finite-volume discretization and admitting unstructured meshes with arbitrarily shaped cells. The numerical advancements are evaluated with canonical simulations of convective planetary boundary layer and strongly (stably) stratified orographic flows, epitomizing diverse aspects of highly nonlinear nonhydrostatic dynamics. The unstructured-mesh solutions are compared to equivalent results generated with an established structured-grid model and observation.
\end{abstract}

Key words: unstructured mesh atmospheric models, nonoscillatory forward-in-time schemes, anelastic equations, orographic flows, planetary boundary layers

PACS:

\section{INTRODUCTION}

Established numerical models for simulating atmospheric flows are typically built on Cartesian grids that have undisputed merits of simplicity and computational economy for homogeneous flows in simple domains. This, however,

* Corresponding Author.

Email address: smolar@ecmwf . int (Piotr K. Smolarkiewicz). 
ceases to be the case for flow problems with large degree of heterogeneity, complex geometry of bounding domains, and multiplicity of scales. In particular for higher resolutions necessary to capture detailed flow features in multi-scale flows, Cartesian grids would require an impractical many-fold increase in the number of grid points. The awareness of limitations of Cartesian grids and a promise of potential benefits of mesh adaptivity, have stimulated interest of the atmospheric community in the development of unstructured-mesh solvers. Historically, studies exploring unstructured meshing for needs of computational meteorology date back to the nineteen sixties [45]. However, a more general and continuous interest in various forms of mesh adaptivity [2] and alternatives to Cartesian mesh models [25] emerged only recently with the advent of multiscale Earth-system modeling. Consequently, because flexible unstructured-mesh models are still relatively new to computational meteorology, they have not yet met the demands of modern operational weather prediction and climate studies, as reviewed in [19]. Further discussion of numerical developments in modeling atmospheric flows on meshes alternative to Cartesian can be found in [38] and references therein.

Factors restricting flexibility of computational meshes may vary. The most common constraints are related to structured connectivities such as “i, j, k" indexing or binary trees. Such structured connectivities are required by discretizations relying on systematic principles for identification of neighboring points. Other limitations - e.g., often utilized in icosahedral mesh schemes - may be introduced by rigid geometrical dependencies on analytical relations used in computations of geometrical metrics. Limitations may also be imposed within discretizations of differential operators in implementations relying on restrictive local mesh regularities, orthogonality, or shape uniformity of cells. Herein, we focus on fully flexible unstructured meshes, naturally accommodating optimal spatial resolution. In particular, for the spatial finite volume integration, we adopt median-dual meshes with edge-based connectivity, specified in Fig. 1 in two dimensions (2D) for clarity. The edge-based connectivity is particularly flexible, as it permits finite volume discretization operating on arbitrary irregular polyhedral cells and is suitable for discretizations of geometrically complex domains. In turn, the median-dual approach benefits the accuracy of calculations on skewed meshes [33]. The presented numerical development is valid for arbitrarily shaped median-dual meshes.

This work builds on the numerical methodologies developed originally for the structured grid model [23] for simulation of atmospheric circulations with anelastic equations on scales from laboratory and wind tunnel $[43,35]$ to global $[30,9]$. The structured grid model is formulated in generalized time-dependent curvilinear coordinates [22,44,31], enabling dynamic mesh adaptivity via continuous mappings [14], and employs unique, nonoscillatory forward-in-time 


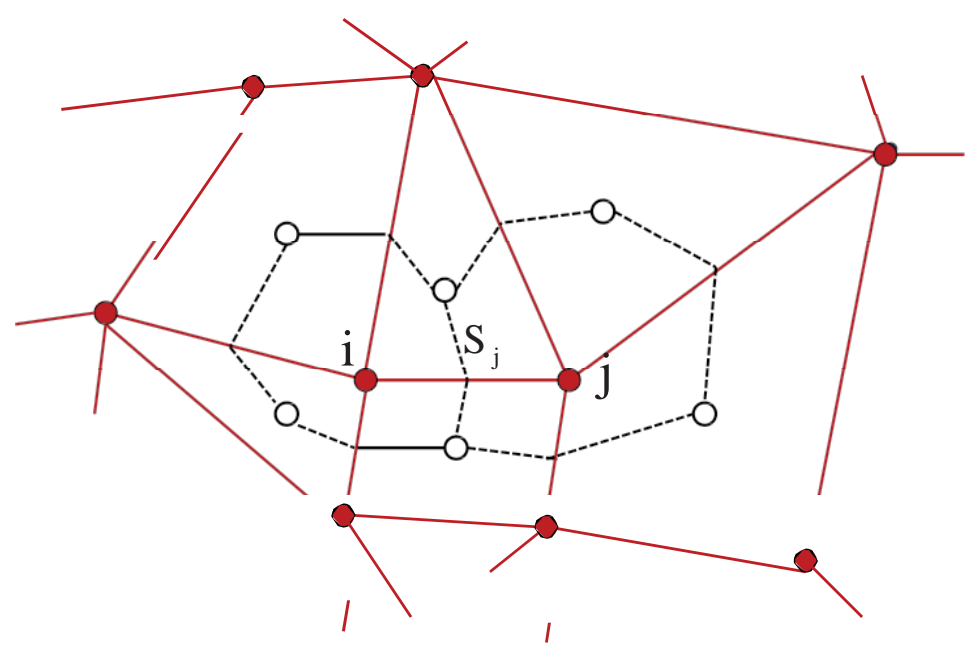

Fig. 1. The edge-based, median-dual approach in 2D. The edge connecting vertices $\mathbf{i}$ and $\mathbf{j}$ of the primary polygonal mesh pierces, precisely in its middle, the face $\mathbf{S}_{\mathbf{j}}$ shared by computational dual cells surrounding vertices $\mathbf{i}$ and $\mathbf{j}$; open circles represent barycenters of the primary mesh, while solid and dashed lines mark primary and dual meshes, respectively.

(NFT) ${ }^{1}$ high-resolution numerics [28,34] based on the MPDATA (for multidimensional positive definite advection transport algorithm) methods; see [34] for a recent overview and comprehensive list of references. To mitigate limitations of rigid connectivity and extend the range of structured-grid model applications, the key methodologies of the NFT model were generalized to unstructured meshes.

In a series of papers a class of the edge-based unstructured-mesh MPDATA schemes $[32,33]$ is derived from first principles, and the corresponding unstructured mesh finite-volume NFT framework is formulated for modeling engineering $[40,36]$ and atmospheric flows $[41,42,38]$. In particular, [41] introduces a class of global hydrostatic models that employ a classical geospherical reference frame with the governing equations cast in the latitude-longitude surfacebased coordinates, but resolve notorious issues associated with meridians convergence near the poles by exploiting the flexibility of unstructured mesh discretization. Next, the development moved onto nonhydrostatic modeling, extending the unstructured-mesh NFT framework to two-dimensional (2D) slabsymmetric, non-hydrostatic mesoscale orographic flows and a range of internal gravity wave phenomena [42,38]. Notably, similarly as in the structured-grid NFT model, the unstructured-mesh nonhydrostatic framework admits various soundproof systems - including the classical incompressible Boussinesq,

1 "Nonoscillatory forward-in-time" labels a class of second-order-accurate twotime-level algorithms built on nonlinear advection techniques that suppress/reduce/control numerical oscillations characteristic of higher-order linear schemes; and it is meant to distinguish these algorithms from classical centeredin-time-and-space linear methods. 
anelastic Lipps-Hemler [16,17] and Durran pseudo-incompressible [6] partial differential equations (PDEs) - thus necessitating the solution of the elliptic Poisson equation implied by the soundproof mass-continuity constraint; see [38] for an exposition. Furthermore, the works [42,38] verified the excellent accuracy of the 2D edge-based approach using benchmarks from both analytic and laboratory results, and comparing unstructured mesh results to the corresponding structured grid model results for simulations of nonhydrostatic mountain waves at weak and strong background stratifications with linear and nonlinear flow response, respectively.

The current paper documents a major development, generalizing previous work to three-dimensional (3D) nonhydrostatic modeling of small-to-mesoscale "dry" atmospheric dynamics. Accounting for the multiphase thermodynamics of atmosphere due to ubiquity of water substance adds complexity on its own and warrants a separate study. Consequently, the paper concentrates on numerical designs pertinent to thermal convection, development of planetary boundary layer (PBL), and flows in complex terrain. A corresponding 3D semi-implicit edge-based unstructured-mesh model is presented and employed in inherently 3D benchmark simulations of evolutionary convective PBL and strongly (but stably) stratified flows past an isolated hill. The results capture well the quantitative aspects of these canonical flow problems and, based on the cumulative experience with numerically akin structured-grid models, indicate that the edge-based model is capable of accurately representing nonhydrostatic flow responses to lower boundary forcings, important for predictability of larger meso- and synoptic-scale systems.

In the following section we present the governing equations underlying the local area nonhydrostatic anelastic model, whereas integration schemes are discussed in section 3. Benchmark simulations are analyzed in section 4, and remarks in section 5 conclude the paper.

\section{GOVERNING EQUATIONS}

The governing equations underlying the local area edge-based model adopt the Lipps-Hemler nonhydrostatic anelastic system [16,17], suitable for simulation of a broad range of atmospheric flows [13,37]. For dry dynamics addressed in this paper, these equations govern the evolution of momentum and entropy, constrained by the incompressible-like mass continuity equation. In a rotating Cartesian reference frame, they can be compactly written as

$$
\frac{d u}{d t}=-\nabla \pi^{\prime}-g \frac{\Theta^{\prime}}{\Theta_{0}}-f \times u^{\prime}+D_{u},
$$




$$
\begin{aligned}
& \frac{d \Theta^{\prime}}{d t}=-u \cdot \nabla \Theta_{e}+D_{\Theta}, \\
& \nabla \cdot \rho_{0} u=0 .
\end{aligned}
$$

Here, vector $u$ denotes the flow velocity, and $\Theta$ is the potential temperature - tantamount to specific entropy via $d s=c_{p} d \ln \Theta$, with $c_{p}$ denoting the specific heat at constant pressure. The differential operators of the total derivative $\mathrm{d} / \mathrm{dt}$ and the nabla $\nabla=\left(\partial_{\mathrm{x}}, \partial_{\mathrm{y}}, \partial_{\mathrm{z}}\right)$ have their generic meaning; so, $\mathrm{d} / \mathrm{dt}=\partial / \partial \mathrm{t}+\mathrm{u} \cdot \nabla$. The Coriolis parameter $\mathrm{f} \equiv 2 \Omega$, where $\Omega$ denotes a constant angular velocity of the rotating reference frame. On the right-hand-side (rhs) of the momentum equation (1), $\pi=\left(p-p_{0}\right) / \rho_{0}$ is a density-normalized pressure perturbation. ${ }^{2}$ Here, the basic (reference) state of the anelastic asymptotic expansion (indicated by the subscript "o") assumes constant stratification $S=d \ln \Theta_{0} / d z \geq 0$ and hydrostatic balance with a constant magnitude of gravitational acceleration $g=(0,0,-g)$; altogether, this defines the background density stratification $\rho_{0}(z)$ [3]. Explicit viscous/diabatic forcings are symbolized with $D$ in all prognostic equations, and can take a variety of forms (e.g., scalar or vector Laplacian, Rayleigh friction, Newtonian cooling, Stokes drag, or suitable combinations of such) depending on the problem at hand. Primes that appear in the momentum and entropy equations denote perturbations with respect to an "ambient" state (indicated by the subscript "e") assumed to be a particular solution of the governing anelastic system. In the elementary case, the ambient state coincides with the basic state. In general, however, resorting to alternative perturbation forms of the governing equations can simplify the design of the initial and boundary conditions as well as enhance the accuracy of calculations in finite-precision arithmetic. Useful examples of ambient states include geostrophically and thermally balanced large-scale flows [30,9],

\section{INTEGRATION SCHEMES}

\subsection{Background}

All prognostic equations of the systems (1)-(3) are of the form $d \psi / d t=R$. Here, $\psi$ symbolizes the potential temperature or its perturbation, and components of the velocity vector; whereas $\mathrm{R}$ denotes the associated right-hand-

$\overline{2}$ Including the density under the gradient operator is a signature of the LippsHemler system [17]; it benefits the conditioning of the elliptic pressure equation [38]. 
sides. Accordingly, by combining $\rho_{\mathrm{o}} \cdot(\mathrm{d} \psi / \mathrm{dt}=\mathrm{R})$ with $\psi \cdot\left(\nabla \rho_{0} \mathrm{u}=0\right)$, the mathematically equivalent conservation-law form can be written as

$$
\frac{\partial \rho_{0} \Psi}{\partial \mathrm{t}}+\nabla \cdot\left(\rho_{0} u \Psi\right)=\rho_{0} R
$$

The nonoscillatory forward-in-time (NFT) algorithm employed to integrate (4) can be written in a compact functional form as

$$
\psi_{i}^{n+1}=A_{i}\left(\tilde{\psi}, v^{n+1 / 2}, \rho_{0}\right)+0.5 \delta R_{i}^{n+1} \equiv \psi_{i}+0.5 \delta t_{i}^{n+1} ;
$$

where $\psi_{\mathrm{i}}^{\mathrm{n}+1}$ is the solution sought at the point $\left(\mathrm{t}^{\mathrm{n}+1}, \mathrm{x}_{\mathrm{i}}\right)$ of a co-located mesh, $\psi \equiv \psi^{\mathrm{n}}+0.5 \delta \mathrm{tR}^{\mathrm{n}}, \mathrm{v} \equiv \rho_{0} \mathrm{u}$, and the normalization by the density $\rho_{0}$ is accounted for within the discrete transport operator A, a shorthand for a finitevolume nonoscillatory two-time level advection algorithm MPDATA [32-34]. ${ }^{3}$ Advecting the auxiliary variable $\tilde{\psi}$ in (5) - reminiscent of Strang splitting [39] - reproduces the trapezoidal-integral structure of the underlying Lagrangian expression $\mathrm{d} \psi / \mathrm{dt}=\mathrm{R}$, and compensates for the first-order error proportional to $\nabla \cdot v R$ characteristic of forward-in-time schemes [28]. Centering in time the advective momentum $v^{n+1 / 2}$ that appears as an argument of $A$ is required to compensate for the first order errors proportional to $\partial \mathrm{v} / \partial \mathrm{t}$ [34]. For the second-order accuracy of the solution in (5), it suffices to provide only a first order accurate estimate of $v_{i}^{n+1 / 2}$. While $O\left(\delta t^{2}\right)$ nonlinear two-time-level predictors can benefit the stability of elastic systems [41], for anelastic equations the linear extrapolation from $v^{n-1}$ and $v^{n}$ is preferred, and used exclusively in this paper, because it assures that $v^{n+1 / 2}$ satisfies the mass continuity equation, provided $v^{n-1}$ and $v^{n}$ do. Furthermore, advecting dependent specific variables $\psi$ with momentum $v$ - rather than dependent density variable $\varphi=\rho_{0} \psi$ with velocity $u$ - assures the compatibility of advection with the discrete anelastic mass continuity constraint, producing no spurious time tendency $\partial_{\mathrm{t}} \psi$ wherever $\psi$ is locally constant; see [14] for an extended discussion.

The overall construct of the scalar NFT algorithm (5) is optimal for the efficacy of second-order-accurate solutions [28,34] as it requires no overhead for compensating the $\sim \nabla \cdot v R$ errors, unlike the compensation in problems with

3 The essence of MPDATA methods is the iterative application of the first-orderaccurate, yet sign-preserving generic upwind scheme, with first iteration providing first-order accurate solution, and subsequent iterations compensating for errors of the preceding iterations. While the basic scheme is second-order accurate and sign preserving for arbitrary flows, options are available that extend MPDATA to full monotonicity preservation, third-order accuracy, and varible sign fields [29,34]. All calculations reported in this paper use the monotone "infinite-gage" variant of MPDATA; cf. section 5.1 in [33]. 
rhs forcings predicted directly at $\mathrm{t}^{\mathrm{n}+1 / 2}$ [9]. Adopting the template (5) to a set of PDEs governing the evolution of a vector of dependent variables lies in approximation of individual forcings $R_{i}^{n+1}=R\left(t^{n+1}, x_{i}\right)+O\left(\delta t^{2}\right)$, customized for a given class of problems. For simplicity of the presentation, in the following we shall first discuss the adaptation of (5) to the non-rotating, adiabatic and inviscid case of (1)-(3), fundamental to modeling buoyant dynamics of thermal convection and gravity waves. The adopted extensions will be highlighted afterwords.

\subsection{Elemental scheme}

At heart of anelastic equations are elliptic boundary value problems (BVPs) dictated by the mass continuity constraint. To outline the formulation of such a BVP we consider an inviscid non-rotating form of the anelastic system (1)(3), and use the symbolic relation (5) to form a system of discrete equations implicit with respect to all dependent variables in the system, as the velocity, pressure, and potential temperature are assumed to be unknown at $n+1$. Assuming a horizontally uniform ambient state $\left(u_{e}(z), \Theta_{e}(z)\right)=$ $\left(u_{e}(z), 0,0, \Theta_{e}(z)\right)$, the respective realizations of (5) for $u, v, w$, and $\Theta$ become

$$
\begin{aligned}
& u=\hat{u}-0.5 \delta t \partial_{x} \pi^{\prime} \\
& v=\hat{v}-0.5 \delta t \partial_{y} \pi^{\prime} \\
& w=\hat{w}-0.5 \delta t \partial_{z} \pi^{\prime}+0.5 \delta t \beta \Theta^{\prime} \\
& \Theta^{\prime}=\hat{\Theta}^{\prime}-0.5 \delta t w \partial_{z} \Theta_{e} .
\end{aligned}
$$

Here $\beta=g / \Theta_{0}$, and all references to the co-located mesh $\left.\right|_{i} ^{n+1}$ have been suppressed for conciseness. Inserting the fourth equation of (6) into the third, regrouping the terms proportional to $w$ on the left-hand side, and dividing the entire equation by the coefficient $\left[1+0.25 \delta t^{2} \beta \partial_{z} \Theta_{e}\right]$ that multiplies $w$, results in the closed form formulae for the update of the velocity components

$$
\begin{aligned}
& u=\hat{u}-C^{x x} \partial_{x} \pi^{\prime} \\
& u=\hat{v}-C^{y y} \partial_{x} \pi^{\prime} \\
& w=\hat{w}-C^{z z} \partial_{z} \pi^{\prime} ;
\end{aligned}
$$

with the modified explicit parts of the solution

$$
\hat{u}=\hat{u}, \quad \hat{v}=\hat{v}, \quad \hat{w}=(\hat{w}+0.5 \delta t \beta \widehat{\Theta})\left[1+0.25 \delta t^{2} \beta \partial_{z} \Theta \mathrm{e}^{-1} ;\right.
$$


and coefficients

$$
C^{x x}=C^{y y}=0.5 \delta t, \quad C^{z z}=0.5 \delta t\left[1+0.25 \delta t^{2} \beta \partial_{z} \Theta_{\Theta}\right]^{-1} .
$$

Having derived (7), the remaining part of formulating the BVP associated with (5) follows straightforwardly. Multiplying all three equations in (7) by $\rho_{\circ}$ and applying the discrete nabla operator $\left(\partial_{x}, \partial_{y}, \partial_{z}\right) \cdot$ consistent with (3) to the resulting momentum vector $\left(\rho_{0} u, \rho_{0} v, \rho_{0} w\right)$ generates the associated Poisson problem

$$
\begin{aligned}
& \frac{1}{\rho_{o}} \partial_{x} \rho_{o}\left(\hat{u}-C^{x x} \partial_{x} \pi^{\prime}\right)+\left[\partial_{y} \rho_{o}\left(\hat{v}-C^{y y} \partial_{y} \pi^{\prime}\right)+\partial_{z} \rho_{o}\left(\hat{w}-C^{z z} \partial_{z} \pi^{\prime}\right)\right. \\
& \equiv \frac{1}{\rho_{o}} \nabla \cdot \rho_{0}(\hat{u}-C \nabla \pi)=0,
\end{aligned}
$$

where $\mathrm{L}=\left(\rho_{0}\right)^{-1} \nabla \cdot\left(\rho_{0} \mathrm{C} \nabla\right)$ and $\mathrm{R}=\left(\rho_{0}\right)^{-1} \nabla \cdot \rho_{0} \hat{\mathrm{u}}$ symbolize the discrete differential operator and explicit counterpart of a sparse linear problem $-(L(\pi)-R)=0$ specified in (10). In this paper, boundary conditions for (10) are either periodic or derive from the Dirichlet boundary conditions for velocity, $n \cdot u_{B}^{n+1}=n \cdot u_{e}$, that imply Neumann boundary conditions for $\pi$, $n \cdot C \nabla \pi_{B}^{\prime}=n \cdot\left(\hat{u}-u_{e}\right)_{B}^{e^{n+1}}$; here subscript ${ }_{B}$ refers to the boundary points, and $\mathrm{n}$ is the outward unit normal to the boundary $\partial \Omega$ of the integration domain $\Omega$. Such a design of the boundary conditions assures the integrability condition ${ }_{\partial \Omega} \rho_{0} n \cdot u^{n+1}=0$ for (10), given ${ }_{\partial \Omega} n \cdot u_{e}=0$. The elliptic problem in (10) is effectively solved to a specified physically-motivated tolerance,

$r_{u} \infty_{\infty} \equiv\left(\delta t / \rho_{0}\right) \nabla \cdot\left(\rho_{0} u\right) \quad \infty \leq \varepsilon$, using the preconditioned generalized conjugate residual algorithm (GCR) reviewed recently in [38]. Given the solution to (10), and thus to (7), updating $\Theta$ from the last equation in (6) completes the elemental scheme.

\subsection{Extensions}

The outlined formulation of the BVP dictated by a forward-in-time model readily extends to flow problems with Coriolis acceleration, Newtonian cooling and Rayleigh friction [22], whereas more cumbersome linear functionals and nonlinear terms can be delegated to the explicit counterpart $R$ by means of outer iteration or its special case the predictor/corrector approach $[9,36,38]$. There is no single principle guiding the degree of the semi-implicitness in a model design, yet semi-implicit schemes are usually selected for computational stability, admitting large time step integrations. In forward-in-time schemes, however, semi-implicit algorithms may be selected equally well for their accuracy; for example, wherever strict time centering is desired for time reversibil- 
ity of a process. The elemental scheme of the preceding section illustrates this point.

In small-to-mesoscale problems, the advective CFL condition typically dictates the model time step, so there is no need for the trapezoidal integral with respect to potential temperature (viz. entropy) fluctuations in (6). In fact, a second-order-accurate yet explicit design (optionally available in the model code) is significantly simpler. Replacing (2) with

$$
\frac{d \Theta}{d t}=D_{\Theta},
$$

and thus integrating homogeneous (4) for the full $\Theta$ using (5) with $\mathrm{R} \equiv 0$ for the elemental problem of section 3.2, replaces the fourth of the equations in (6) with $\Theta^{\prime}=\hat{\Theta}-\Theta_{e}$ subsequently used in the buoyancy term in the third equation of (6). The resulting explicit parts of the solution in (8) and the coefficients in (9) become then, respectively,

$$
\begin{aligned}
& \hat{u}=\hat{u}, \quad \hat{v}=\hat{v}, \quad \hat{w}=(\hat{w}+0.5 \delta t \beta \hat{\Theta}) ; \\
& C^{x x}=C^{y y}=C^{z z}=0.5 \delta t .
\end{aligned}
$$

Notwithstanding the apparent simplicity of the explicit scheme, there are several important accuracy benefits of the semi-implicit scheme [30]. In particular, transporting only fluctuations of $\Theta$, while retaining the convective derivative of the ambient state on the right-hand side, assures conservation of the fluctuations with accuracy to round-off error (section 3a [30]), tantamount to preventing dilution of the background stratification due to the implicit viscosity of non-oscillatory advection. Furthermore, when solutions are sought in terms of fluctuations about a metastable ambient profile [8], the semi-implicit algorithm enables dynamic equilibria that might have been unreachable otherwise. On a technical side, the semi-implicit algorithm extends the stencil of the linear operator in (10), thus suppressing its null space resulting from a co-located mesh. Because of these benefits, the implicit scheme is standard in our models, and it has been employed in all calculations presented in section 4 of the paper.

Extending the semi-implicit scheme in order to center in time the Coriolis terms in (1) can be laborious. Furthermore, accounting for time-centering of Rayleigh friction and Newtonian cooling in (1) and (2), respectively, adds further complexity to the resulting Poisson problem. However, implicit integrations of the Coriolis terms benefit accuracy of the long-term global integrations of planetary and stellar climates $[30,8]$, whereas the implicitness of the Rayleigh friction and Newtonian cooling is a key in simulating complex boundaries of internal structures via immersed boundary approach [12] 
- neither of which are addressed here. Consequently, the elemental scheme of section section 3.2 is extended to the full set of governing equations (1)(3) using explicit schemes. Specifically, the dissipative/diabatic forcings, D terms in (1)-(2), are integrated explicitly to $O\left(\delta t^{2}\right)$ by extending $\tilde{\psi}$ in (5) as $\tilde{\psi} \equiv \psi^{\mathrm{n}}+0.5 \delta \mathrm{tR} \mathbf{i}^{\mathrm{n}}+\delta \mathrm{tR} v^{\mathrm{n}}$, with $\mathrm{Ri}$ and $\mathrm{Rv}$ denoting inviscid/adiabatic and viscous/diabatic counterparts of $R=R \mathbf{i}+R v$. The Coriolis terms are integrated to $\mathrm{O}\left(\delta \mathrm{t}^{3}\right)$, using outer iteration for the augmented problem $(7)-(10)$, such that:

$$
\begin{aligned}
& u^{v}=\check{u}-\left.C \nabla \pi\right|^{v}, \quad \check{u} \equiv \hat{u}-0.5 \delta t f \times\left(u^{v-1}-u_{e}\right) ; \\
& \nabla \cdot\left(\rho_{0} u^{v}\right)=0 ;
\end{aligned}
$$

where $v$ counts outer iterations. Because the time scale of Coriolis acceleration $(24 \mathrm{~h})$ is orders of magnitude larger than mesoscale $\delta t \sim O(10) \mathrm{s}$, the scheme converges rapidly, in a few outer iterations [36]. Additional solutions of the elliptic problems are cost-wise minuscule, because the work of the solver decreases dramatically beyond the first iteration; see [38] for a related discussion.

\subsection{Highlights of spatial discretization}

The derivations and details of median-dual unstructured mesh discretization of differential operators entering MPDATA schemes, symbolized with A in (5), are provided in $[32,33]$ and $[40]$ assuming an arbitrary 3D arrangement. For completeness, here we highlight a key tool for designing discrete differential operators for edge-based control-volume schemes.

Figure 2 marks a face $S_{j}$ of an arbitrary computational cell containing vertex $\mathbf{i}$, together with the edge connecting vertex $i$ with one of its immediate neighbors $\mathbf{j}$; there are I(i) edges connecting the vertex $\mathbf{i}$ with its neighbors; cf. Fig. 1. $S_{\mathbf{j}}$ refers both to the face per se and its surface area. For a differentiable vector field $A$, the Gauss divergence theorem $-\Omega_{\Omega} \nabla \cdot A={ }_{\partial \Omega} A \cdot n-$ applied over the control volume $V_{i}$ surrounding vertex $i$ leads to

$$
\nabla_{i} \cdot A={\frac{1}{V_{i}}}_{j=1}^{l(i)} A_{j}^{\perp} S_{j} .
$$

Equation (15) is exact given $\nabla_{i} \cdot A$ is interpreted as the mean value of $\nabla \cdot A$ within the volume $V_{i}$, while $A_{j}^{1}$ is interpreted as the mean normal component of the vector $A$ at the cell face $S_{\mathbf{j}}$. The approximation begins with specifying $A_{\perp}^{\perp}$ in terms of data available on the mesh; i.e., in terms of mean values of the field within the control volumes $V_{i}$ and $V_{j}$. One elementary example is

$$
A_{j}^{\perp}=0.5 n_{j} \cdot\left[A_{i}+A_{j}\right],
$$




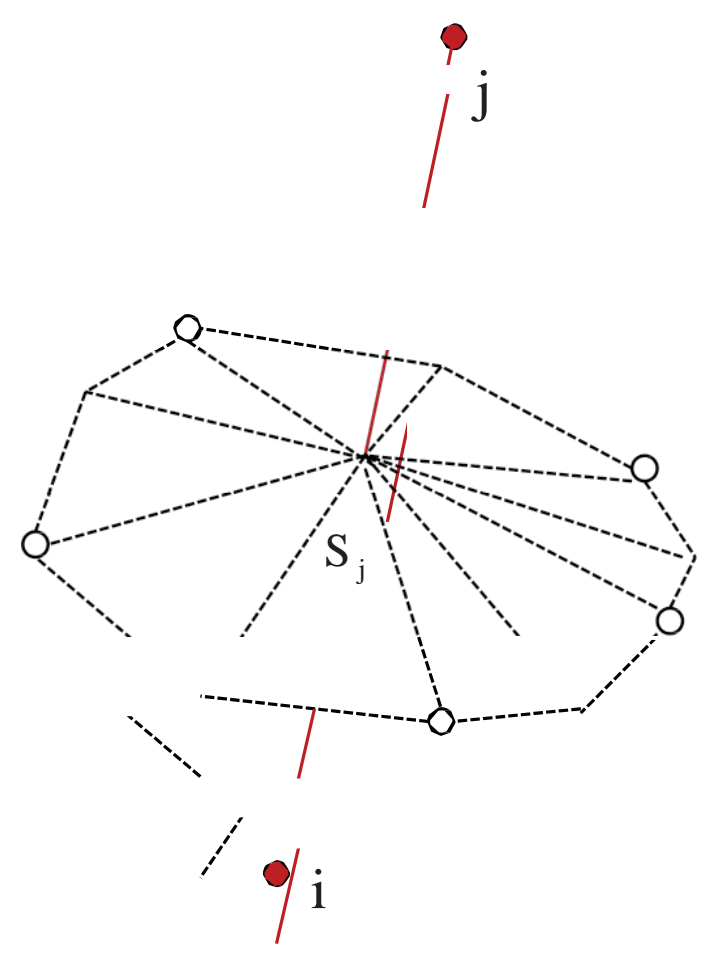

Fig. 2. The edge-based median-dual approach in 3D. The edge connecting vertices $\mathbf{i}$ and $\mathbf{j}$ of the primary mesh pierces (at the edge center) the face $S_{\mathbf{j}}$ of a computational (dual) cell surrounding vertex $\mathbf{i}$; open circles represent barycenters of polyhedral cells surrounding the edge. Dashed lines mark a fragment of the dual mesh; cf. Fig 1.

where $\mathrm{n}_{\mathbf{j}}$ is a mean outward unit normal to the face $\mathrm{S}_{\mathbf{j}}$. Partial derivatives $\partial \Phi / \partial x^{\prime}$ of a scalar field $\Phi$ with subscript $\mathbf{I}=1,2$ or 3 referring to Cartesian coordinates $x, y$ or z, respectively, can also be interpreted in terms of the Gauss theorem (by representing the derivative as the divergence of the augmented vector field $\Phi \nabla x^{\prime}$ ) as, e.g.,

$$
{\frac{\partial \Phi}{\partial x^{\prime}}}_{i}={\frac{1}{V_{i}}}_{j=1}^{l(i)} 0.5\left(\Phi_{i}+\Phi_{j}\right) S_{j}^{l}
$$

where $S_{j}^{\prime}$ denotes the Ith component of the oriented surface element $S_{j}=S_{j} n_{j}$ of the face at the $\mathrm{j}$ th edge.

\section{RESULTS}

In the following we discuss results of the unstructured-mesh model described in the preceding sections applied to address distinct classes of canonical atmospheric mesoscale flows in 3D. The first example quantifies the model fidelity in simulation of convective planetary boundary layer (PBL) over flat terrain. By contrast, the second example addresses influence of complex terrain on 
atmospheric motions in simulation of a strongly stratified flow past a steep isolated hill. Both problems are nonhydrostatic, highly nonlinear, and wellstudied in the literature. The PBL flow is turbulent and stochastic. It typifies the response of an initially stagnant fluid to a diabatic and frictional surface forcing in the lowest $500 \mathrm{~m}$ of the Earth atmosphere. The orographic flow spans both linear and nonlinear flow regimes. Near field solution is characterized by an abundance of complex, yet coherent structures, whereas aloft the flow transitions to a linear gravity wave response. Both benchmarks represent viable elements of nonhydrostatic dynamics affecting predictability of natural weather at larger meso and synoptics scales. For instance, dissipative effects within the planetary boundary layer are important for developing convective structures aloft [20], the interaction of which with deep gravity wave modes in the free troposphere can modulate the organization of the cloud clusters at larger scales $[10,1]$. The finite-amplitude effects related to topographically forced stably stratified flows, such as wave breaking and downslope windstorms or more generally mountain-wave induced turbulence, present obvious challenge for mesoscale weather predictability [5].

\subsection{Convective boundary layer}

We have adapted the numerical experiment from [18], which was one of the first systematic demonstrations of the implicit large-eddy-simulation (ILES) with MPDATA based NFT schemes. The anelastic equations (1)-(3) assume nonrotating Boussinesq limit with constant reference profiles $\Theta_{0}(z)=\Theta_{0}$ and $\rho_{0}(z)=\rho_{0}$. The growth of the convective boundary layer is driven by a prescribed diabatic source in (2), $\mathrm{D}_{\odot}=-\mathrm{dH} / \mathrm{dz}$, with a heat flux specified as $H(z)=H_{0} \exp (-z / \lambda)$, where surface flux $H_{0}=0.01 \mathrm{Kms}^{-1}$ and the vertical scale $\lambda=25 \mathrm{~m}$. The effect of surface friction in (1) is parameterized similarly as $D_{u}=-d_{T} / d z$, with stress $\mathrm{T}=\mathrm{T}_{0} \exp (-z / \lambda)$, where surface stress $\mathrm{T}_{\mathrm{o}}=-\mathrm{C}_{\mathrm{d}} \quad \mathrm{u}_{\mathrm{o}} \quad \mathrm{u}_{0}$, and drag coefficient $\mathrm{C}_{\mathrm{d}}=0.1$. Because these diabatic/viscous forcings quickly decay with height, they only parametrize nearsurface effects; whereas subgrid-scale modeling aloft is delegated to dissipative properties of MPDATA [23,20].

The model domain, $L x \times L y \times L z=3200 \times 3200 \times 1500 \mathrm{~m}^{3}$, is periodic in the horizontal, and a stress-free rigid-lid boundary is assumed at the top. Gravity wave absorbers attenuate the solution toward ambient conditions with the inverse time scale $\alpha \geq 0$, which increases linearly from zero at the distance $0.5 \mathrm{~km}$ below the boundary to $1020^{-1} \mathrm{~s}^{-1}$ at the boundary. Technically, the absorbers enter the governing equations as respective contributions $-\alpha\left(u-u_{e}\right)$ and $-\alpha \Theta$ to $D_{u}$ and $D_{\odot}$ terms in (1) and (2), with $\alpha>0$ only in vicinity of the upper boundary. As an initial condition, a $500 \mathrm{~m}$ deep well-mixed ambient layer is prescribed, with a constant temperature $\Theta_{e}(z)=\Theta_{0}=300 \mathrm{~K}$. Aloft, 
$\Theta_{e}(z)=\Theta_{0}\left[1+z N^{2} / g\right]$ and the Brunt-Väisällä frequency $N=10^{-2} s^{-1}$ define stable stratification $S=N^{2} / g$. The ambient wind field $u_{e}=0$. The initial conditions are generated by randomly perturbing $\Theta_{e}$ and $w$, and then finding the potential flow consistent with mass continuity in (3). At the surface the white-noise perturbation has an amplitude of $0.001 \mathrm{~K}$ for $\Theta$ and $0.2 \mathrm{~ms}^{-1}$ for $\mathrm{w}$, which decrease linearly with height to zero at the top of the mixed layer. Simulations were run for $15000 \mathrm{~s}$. A statistically stationary state is reached after about $9000 \mathrm{~s}$, which is equivalent to about 8 large-eddy turnover time scales $t^{*}$. This time scale is the ratio of the inversion height $z_{i}$ to the convective velocity scale $\mathbf{w}^{*}$. The inversion height is defined as that height where the horizontally averaged Reynolds heat flux is most negative. The convective velocity scale is defined as $\mathrm{w}^{*}=\left(\mathrm{g} / \Theta_{\mathrm{o}} \mathrm{z} \mathrm{H}_{0}\right)^{1 / 3}$; for completeness, a convective temperature scale and the eddy turnover time scale are defined as $\mathrm{T}^{*}=\mathrm{H}_{\delta} \mathrm{W}^{*}$ and $\mathrm{t}^{*}=\mathrm{z}_{\mathrm{i}} / \mathrm{w}^{*}$, respectively.

Three basic simulations were performed for this study. The reference simulation, Run R uses the structured-grid code [23] with $\mathrm{Nx} \times \mathrm{Ny} \times \mathrm{Nz}=$ $64 \times 64 \times 51$ regularly distributed points of a Cartesian grid. Two simulations conducted with the edge-based code, Run $G$ and Run $T$ use, respectively, the Cartesian grid of Run $R$ and the unstructured mesh. The primary unstructured mesh consists of $\mathrm{Nxy} \times \mathrm{Nz}=5228 \times 50$ triangular based prisms, stacked in $50, \delta z=30 \mathrm{~m}$ thick, layers. The corresponding dual mesh, used in numerical integration, is constructed as outlined in Figs. 1 and 2 by joining barycenters, faces and edges of the primary mesh prisms surrounding vertices "i" and "j". Figure 3 displays the instantaneous vertical velocity field at the end of the Run T, organized into characteristic, albeit irregular, RayleighBénard cells [20]. The convective scales for the three runs are collected in Table 1 and compared with Runs E and I of [18] that correspond to calculations with excluded/included explicit subgrid-scale model; i.e, ILES versus LES. The current Runs R, G and T all evince the same characteristic integral scales. They agree with the ILES Run E of [18] to within 5\%. This discrepancy is attributed to (apparently minor) differences between the referenced $E$ run and the current simulations, including free-slip versus partial-slip surface boundary conditions discussed in the opening paragraph of the current section, and explicit versus implicit scheme for the buoyancy forcing discussed in section 3.3. Consequently, the exact correspondence between the current ILES experiments and the equivalent calculation in [18] is not expected. Noteworthy, the current ILES agrees to within 3\% with the LES Run I of [18], which is due to the parameterization of surface friction accounted for in Runs $\mathrm{R}, \mathrm{G}$ and $\mathrm{T}$.

The integral scales in Table 1 appear to validate the turbulence simulations using the edge-based model. However, this sole validation may be insufficient, because only the inversion height $z_{i}$ is measured independently and the other three scales derive from it. To better assess the quality of the edge-based sim- 

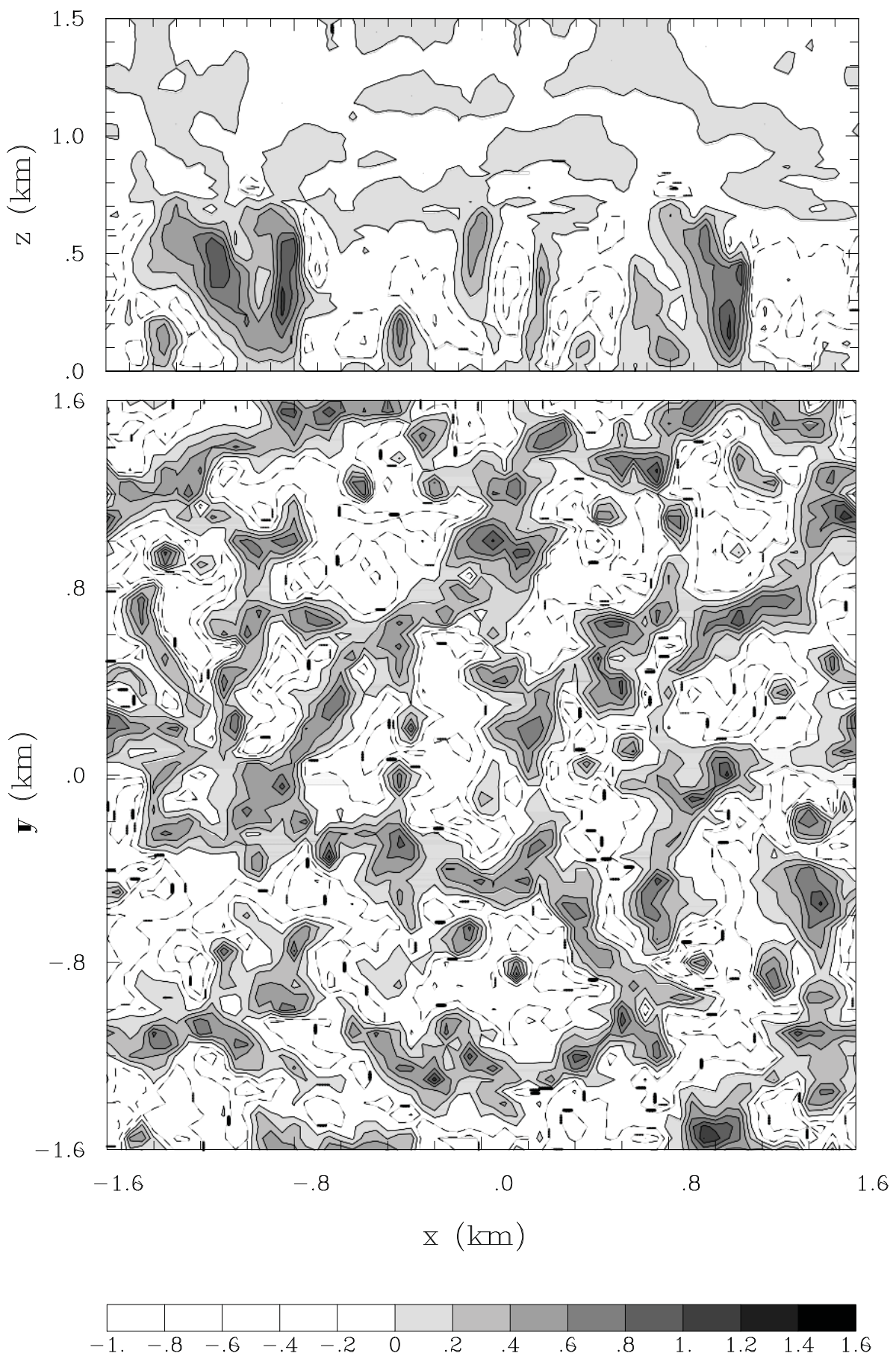

Fig. 3. Vertical velocity in central $x z$ cross section (top) and the horizontal plane at $z=150 \mathrm{~m}$ (bottom); an instantaneous solution for triangular prismatic mesh is shown after $\sim 13$ eddy turnover times; dashed contours indicate negative values.

ulations, we compare the normalized vertical profiles of three resolved second moments generated in the three simulations - heat flux $(\Theta \mathrm{w}) / \mathrm{H}$, temperature variance $\left(\Theta^{\prime}\right) /\left(T^{*}\right)^{2}$, and vertical velocity variance $\left(w^{\prime} w^{\prime}\right) /\left(w^{*}\right)^{2}$, where primes denote deviation from the horizontal average $(\cdots)$. The moments shown 
Table 1

Convective scales for simulations using different models and meshes

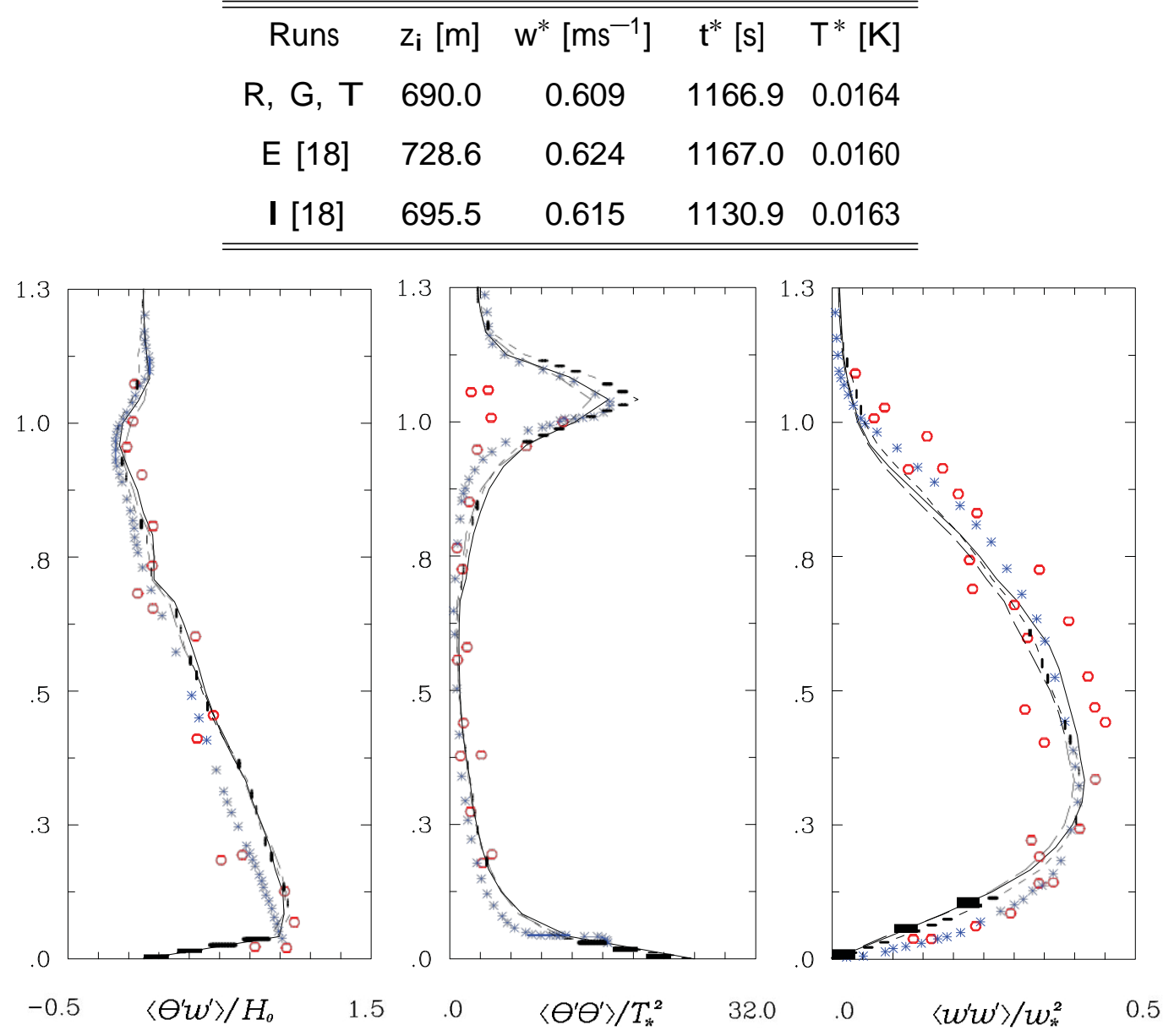

Fig. 4. Vertical profile of dimensionless resolved heat flux, and variances of temperature as well as vertical velocity in Runs $T$ (solid), G (long dashes), and R (short dashes), with dimensionless height $z / z_{\mathbf{i}}$ on the ordinates; blue crosses denote LES result of [24], and red circles represent field and laboratory data.

in Figure 3 are compared with the LES simulations of Schmidt and Schumann [24], and with field [15] and laboratory data [4] superimposed by Schmidt and Schumann. Generally, the profiles generated with the NFT codes match closely each other and agree within the data scatter with the LES calculations of Schmidt and Schumann [24]. An analogous analysis of other moments (not shown) demonstrates similar agreement.

\subsection{Stratified flow past a steep isolated hill}

This example concerns the flow of an anelastic fluid with uniform environmental wind profile $\mathrm{u}_{\mathrm{e}}\left(\mathrm{z}_{\mathrm{c}}\right)=\mathrm{U}=5 \mathrm{~m} \mathrm{~s}^{-1}\left(\mathrm{v}_{\mathrm{e}} \equiv \mathrm{w}_{\mathrm{e}} \equiv 0\right)$ and constant Brunt-Väisällä frequency $N=10^{-2} \mathrm{~s}^{-1}$ past an axially-symmetric cosine hill 
$h(x, y)=h_{0} \cos ^{2}(\pi r / 2 L)$ if $r=\left(x^{2}+y^{2}\right)^{1 / 2} \leq L$ ( $h=0$ otherwise). The hill parameters are $h_{0}=1.5 \cdot 10^{3} \mathrm{~m}$, and $\mathrm{L}=3 \cdot 10^{3} \mathrm{~m}$. The model domain spans $5 \mathrm{~L} \times 4 \mathrm{~L} \times 2 \mathrm{~L}$, respectively, in $x, y$, and $z$. All the results are shown after $\mathrm{t}=1200 \mathrm{~s}$, extending over two advective time scales $\mathrm{T}=\mathrm{L} / \mathrm{U}$ when the main features of the solution have already been established. While the boundary conditions are rigid in $\mathrm{x}, \mathrm{y}$ and $\mathrm{z}$, the gravity-wave absorbers near the upper and lateral streamwise boundaries attenuate the solution toward environmental profiles with absorbing coefficient increasing linearly from zero at the distance $L / 2$ from the boundary to $150^{-1} \mathrm{~s}^{1}$ at the boundary. The initial condition is the potential flow.

This particular choice of environmental profiles and the height of the mountain results in the interesting (in the area of stably stratified flows past complex terrain) fluid regime frequently referred to as a low Froude number flow. Here the Froude number $\left(\mathrm{Fr} \equiv \mathrm{U} / \mathrm{Nh}_{\circ}\right)$ equals $1 / 3$. The distinguishing features of such flows include the separation and reversal of the lower upwind stream, and the formation of intense vertically-oriented vortices on the lee side of the hill $[11,27,7]$; whereas the flow aloft transitions to the linear gravity wave response [26]. The choice of the flow parameters and hill geometry makes the entire problem essentially nonhydrostatic and comparable to laboratory results of [11], $h_{0} / L \sim O(1)$ and Rossby number $R o=U / L f_{z} \mathbf{p} \infty$, while addressing key aspects of natural flows past large isolated mountains such as the big island of Hawaii, $h_{0} / L \sim O(0.1), R o \approx 3$.

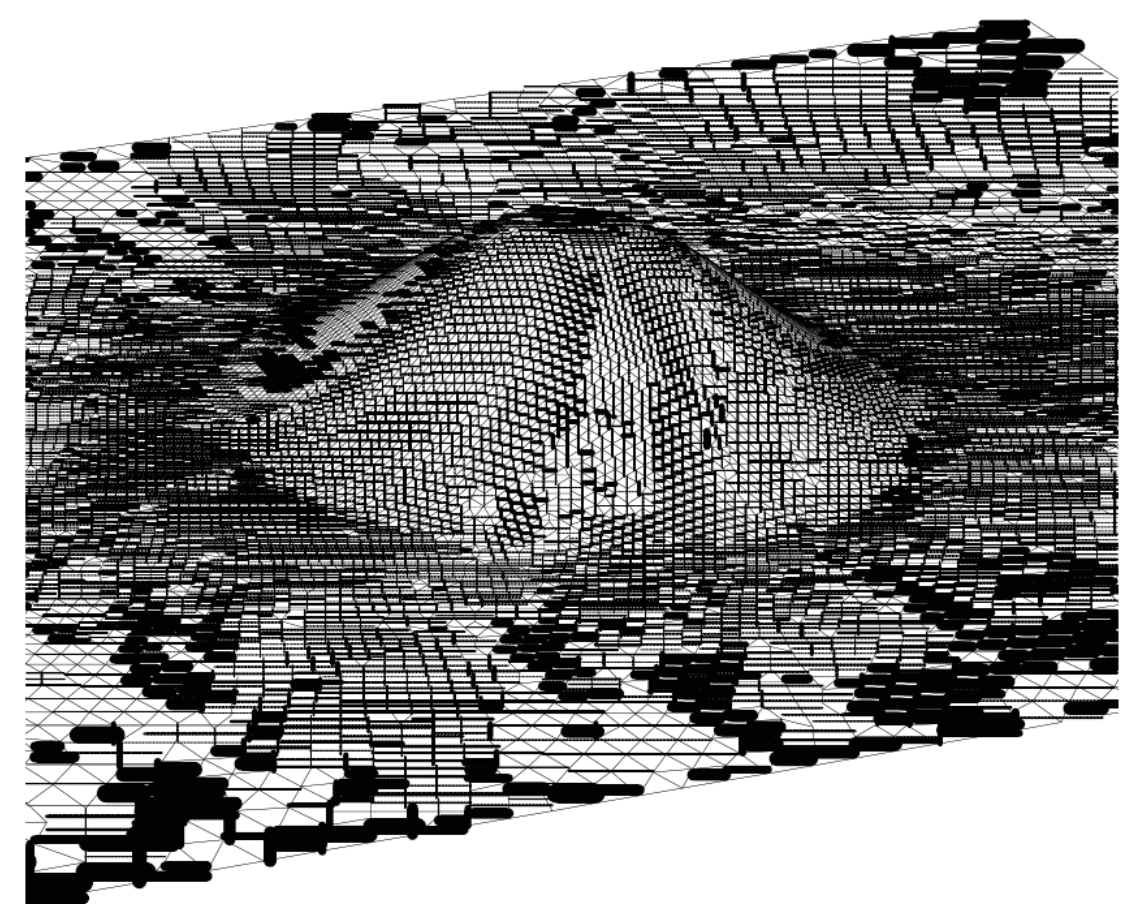

Fig. 5. A fragment of the horizontal primary mesh at the hill's surface, $k=1$ in (18), used in simulations with the edge-based code. 
The solution obtained with the edge-based code uses the unstructured mesh, constructed as follows. First a horizontal triangular mesh is generated that uses varying resolution ranging from $450 \mathrm{~m}$ at the boundaries to ca. $100 \mathrm{~m}$ in the hill vicinity, Fig. 5. This triangular mesh constitutes a base of the $\mathrm{N} x y \times \mathrm{Nz}=11353 \times 61$ point mesh with 60 layers of distorted prisms, stacked in the vertical according to:

$$
z_{i, k}=(k-1) \delta z \quad 1-\frac{h_{i}}{H}+h_{i},
$$

where $\mathbf{i}=1,11353$ numbers horizontal locations on the mesh, $h_{i}$ is the hill elevation at the ith horizontal location, $k=1,61$ denotes levels of cell faces bounding a kth prism in the vertical, $\mathrm{H}=2 \mathrm{~L}$ is the vertical extent of the domain, and $\delta z=100 \mathrm{~m}$. Integrations are performed on the corresponding dual mesh, consisting of arbitrary polyhedra. As discussed in section 4.1, the dual mesh is formed by connecting centers of edges with centers of adjusted prism faces and their barycenters in a manner illustrated in Figs. 1 and 2. The solution using the edge-code is compared with the equivalent solution using the structured-grid code [23] employing regular grid of $\mathrm{Nx} \times \mathrm{Ny} \times \mathrm{Nz}=$ $152 \times 121 \times 61$ points and continuous mapping from Cartesian to computational space, $(\mathrm{x}, \mathrm{y}, \mathrm{z}) \rightarrow(\mathrm{x}, \mathrm{y}, \mathrm{z})$, according to:

$$
\mathrm{x}=\mathrm{x}, \quad \mathrm{y}=\mathrm{y}, \quad \text { and } \mathrm{z}=\mathrm{H} \frac{\mathrm{z}-\mathrm{h}(\mathrm{x}, \mathrm{y})}{\mathrm{H}-\mathrm{h}(\mathrm{x}, \mathrm{y})} ;
$$

see [44] for a discussion. Noteworthy, if in the edge-based code a $\mathrm{Nx} \times \mathrm{Ny}$ Cartesian mesh were to constitute a base of the 60 layers of prisms, the resulting mesh would correspond to a geometric visualization of products of the uniform grid increments $\delta \mathrm{x}, \delta \mathrm{y}, \delta \mathrm{z}$ and appropriate metric coefficients employed in the structured-grid model; cf. Fig. 3 in [38].

Figure 6 conveys the key features of a nonrotating 3D flows with $\mathrm{Fr}<1$. The solution using the structured-grid model is shown in the central $x z$ vertical plane, together with the surface flow as it would be perceived via the ground based measurements. Flow vectors in the two panels are superimposed on the contours of vertical velocity. The upper panel shows a turbulent wake in the lee, with the characteristic gravity-wave response aloft. Lower panel shows two large coherent eddies in the lee, and the splitting of the lower windward stream. This intricate flow structure is due to relative height of the hill so large, that the incoming flow up to $\mathrm{z}_{\mathrm{c}} \approx(1-\mathrm{Fr}) \mathrm{h}_{\circ}$ lacks kinetic energy required to climb over the hill and is forced to deflect around. The flow above $\mathrm{z}_{\mathrm{G}}$ (a dividing streamline height [11]) thus appreciates a reduced mountain with height $h_{r} \approx F r h_{0}$ and is susceptible to linear like gravity-wave response.

Figure 7 complements quantitative highlight of the flow in Fig. 6 with a per- 

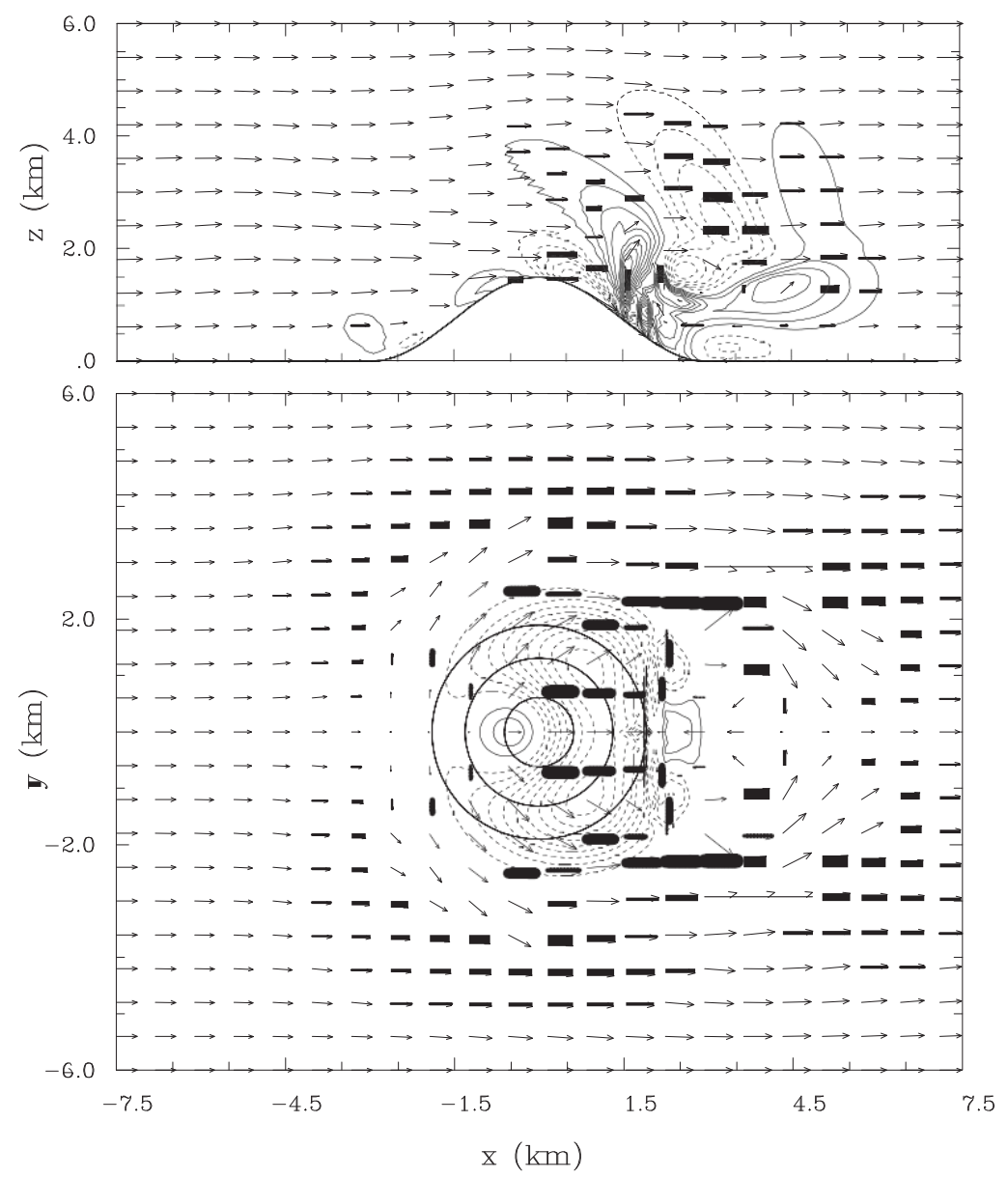

Fig. 6. Vertical velocity in central $x z$ cross section (top) and along the lower boundary $z=h(x, y)$; an instantaneous solution for structured-grid model [23] with $\mathrm{Fr}=1 / 3$, Ro $\boldsymbol{\Gamma} \infty$ is shown after two advective time scales $\mathrm{T}=\mathrm{L} / \mathrm{U}$. Positive and negative values are displayed with solid and dashed contours, respectively, with the interval of $0.5 \mathrm{~m} / \mathrm{s}$, and zero contour lines are not shown; vectors arrows at the inflow boundary correspond to ambient flow $U=5 \mathrm{~ms}^{-1}$, and isolines of hill in the bottom panel are displayed with $500 \mathrm{~m}$ increment starting at $\mathrm{z}=500 \mathrm{~m}$.

spective display of isentropic surfaces originating far upstream, respectively from bottom up, at $z=500,1500$, and $2500 \mathrm{~m}$. This display already employs the edge-based solution, the close comparability of which with the structuredgrid result is documented next.

Figure 8 compares the results generated with the edge-based code to the reference solution obtained with the structured-grid model [23]. The top panel in Fig. 8 corresponds to the top panel of Fig. 6. While the result in Fig. 6 displays directly the result generated in the transformed space (using suitable mapping inside the graphic program), the edge-based solution was first interpolated to the Cartesian grid, and then plotted on it while masking the extrapolated values below the hill's surface. For the masking purpose the hill displayed in the top panel of Fig. 8 assumes the same functional relation, $h=h_{0} \cos ^{2}(\pi r / 2 L)$, 


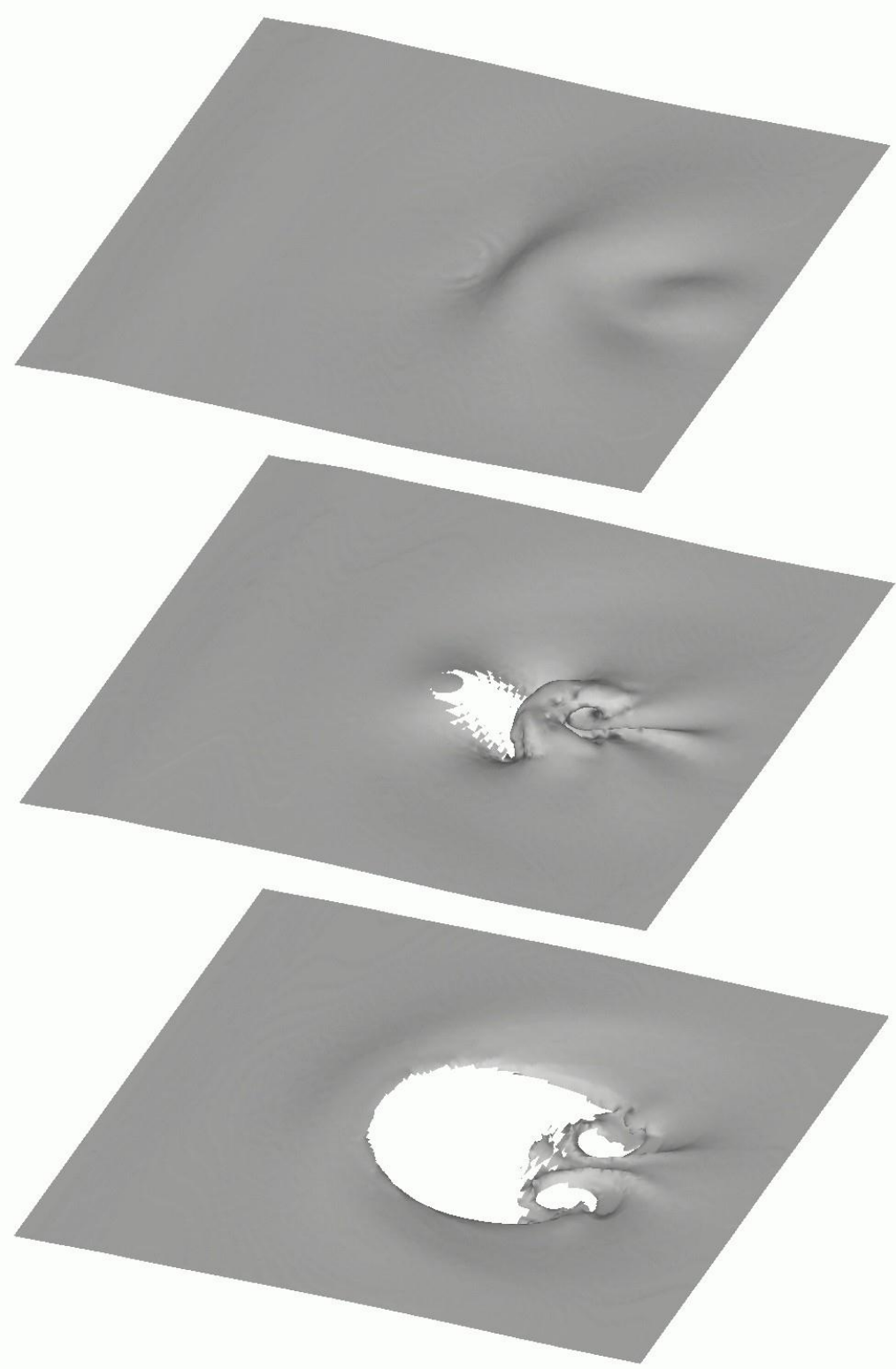

Fig. 7. Isentropes for the edge-based solution with undisturbed heights $z=500,1500$, and $2500 \mathrm{~m}$, from bottom up respectively.

but with slightly elevated amplitude $h_{0}=h_{0}+\delta z$. The central panel of Fig. 8, shows contours of vertical velocity together with the flow arrows in the $x y$ plane at $z=h_{0} / 3$. The lower panel of Fig. 8 shows corresponding solution generated with the structured-grid codes. In spite the differences of the graphics design, flow nonlinearity, and different spatial discretizations the two solution match closely each other. Furthermore, the gravity-wave responses aloft shown for the both solutions in Fig. 9 are virtually the same. While similar high quality results were obtained with both the Cartesian and edge-based solvers, the refinement easily implemented with unstructured meshes allowed for the edge-based solution to be achieved with $38 \%$ reduction in the number 


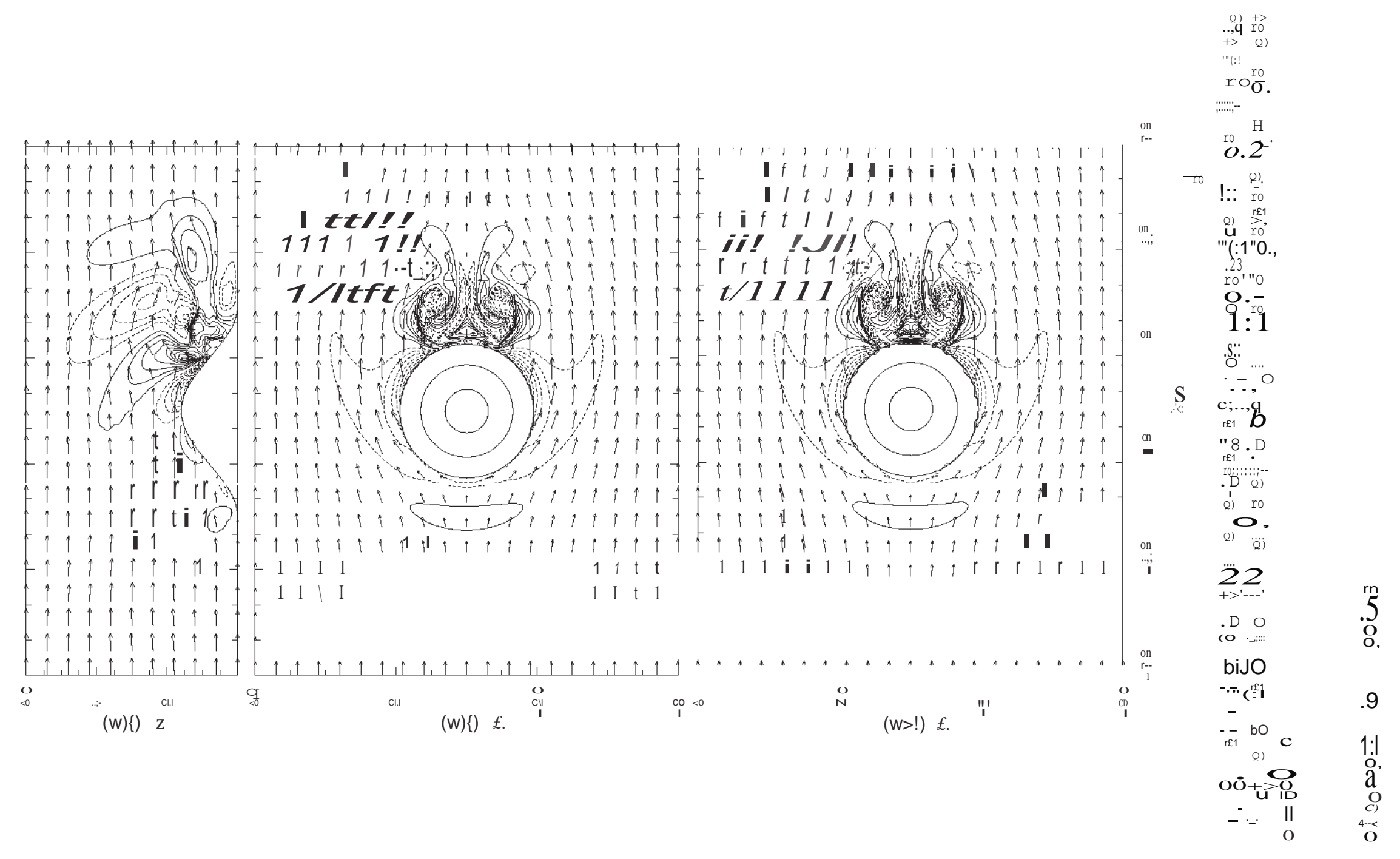




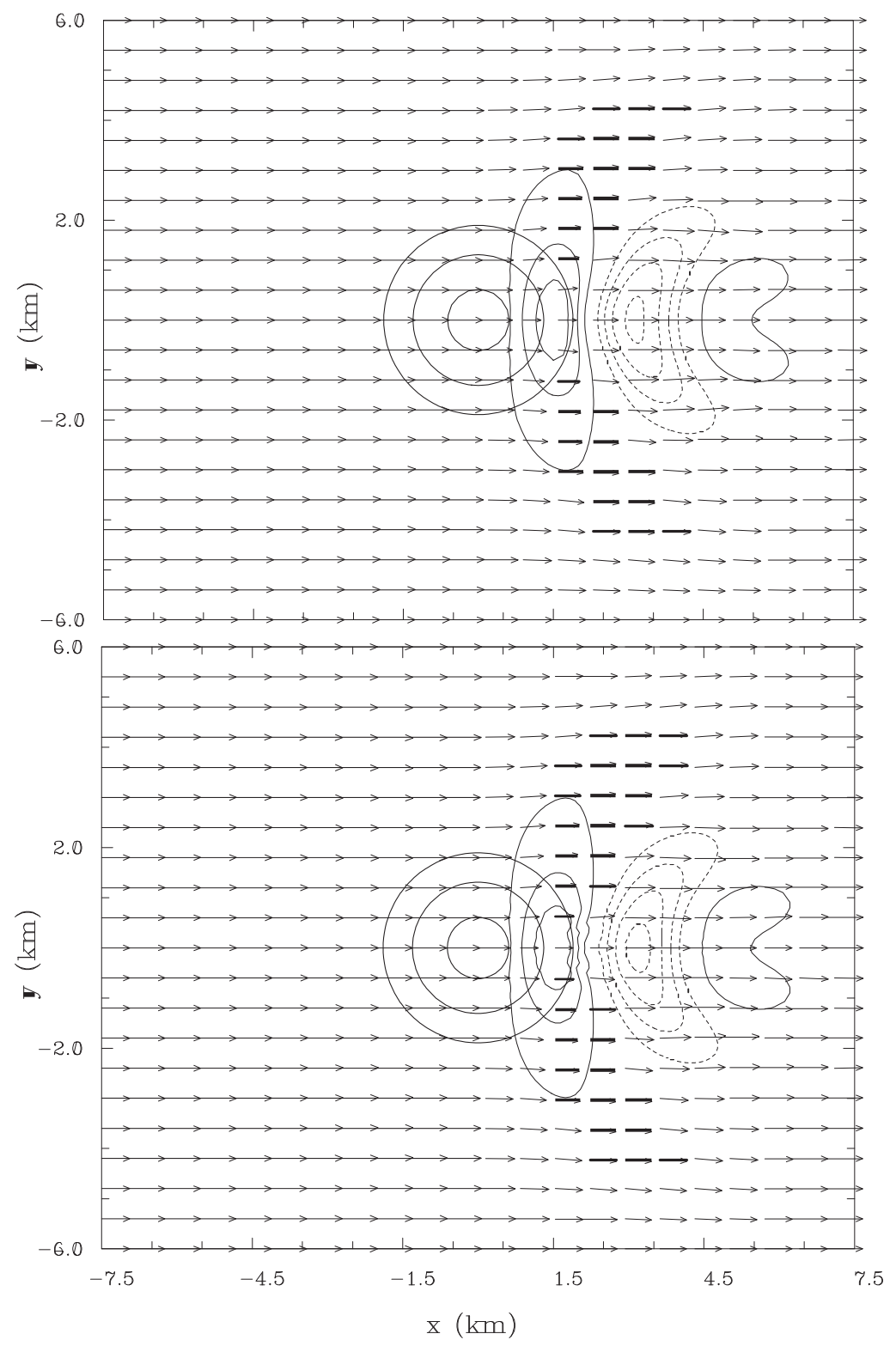

Fig. 9. As in Figs. 6 and 8 but for the solutions $x y$ horizontal planes at $z=2500 \mathrm{~m}$, generated with the edge-based model (top) and structured-grid model (bottom).

Figure 10 contrasts the results in Figs. 6-9 with the rotating flow solution at $\mathrm{Ro} \approx 3$ generated with the edge-based model, assuming the $20^{\circ} \mathrm{N}$ latitude and the planetary rotation 10 times of the Earth. The corresponding results for the structured-grid model are not shown, as they are qualitatively close. However, the differences between the edge-based and structured-grid solutions are more apparent than in the nonrotating case, especially in the turbulent wake seen in the central panel of Fig. 10 at the onset of shedding. Because the ambient flow is geostrophically balanced, the Coriolis force in (1) acts only on the velocity perturbations, which tend to diminish with height. Consequently, the rotational effects also decay with height, producing the gravity wave response 

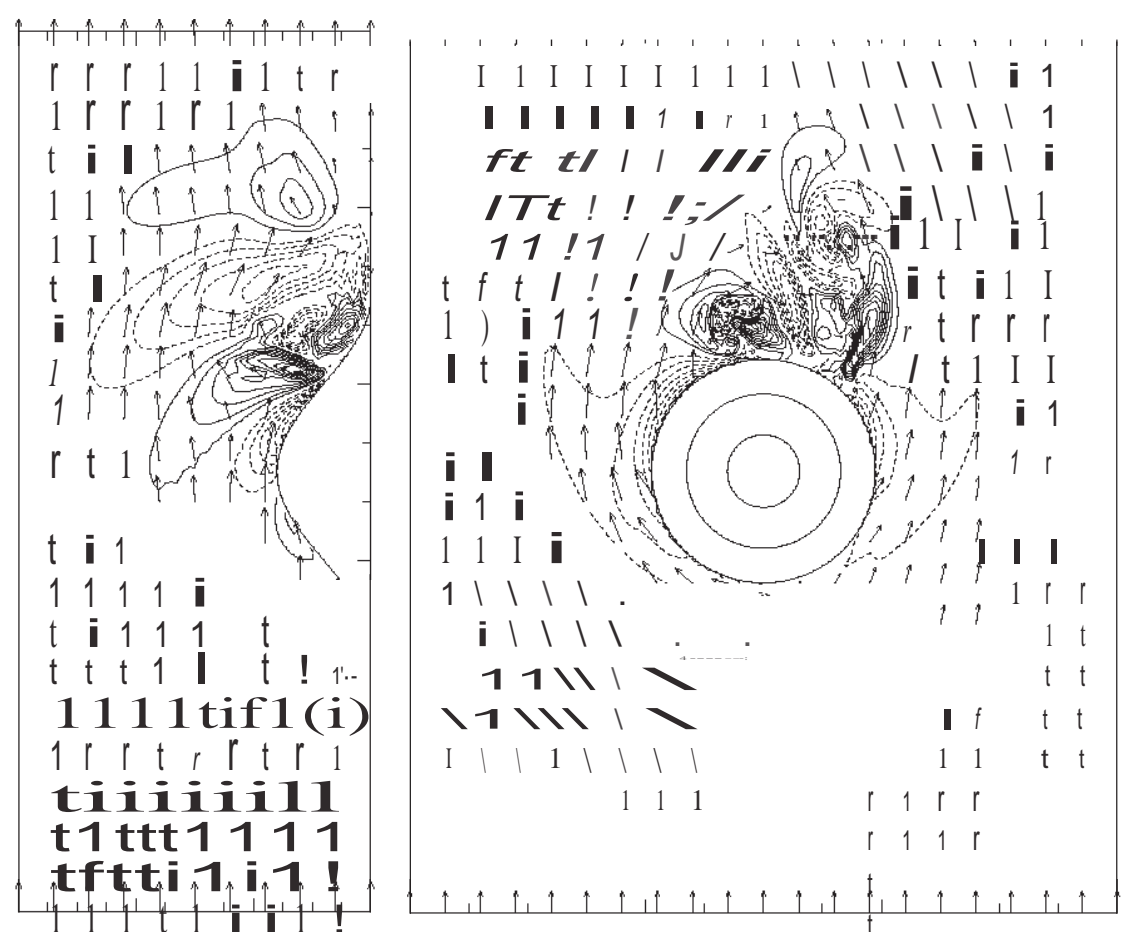

이잉

a

. .0

Cil

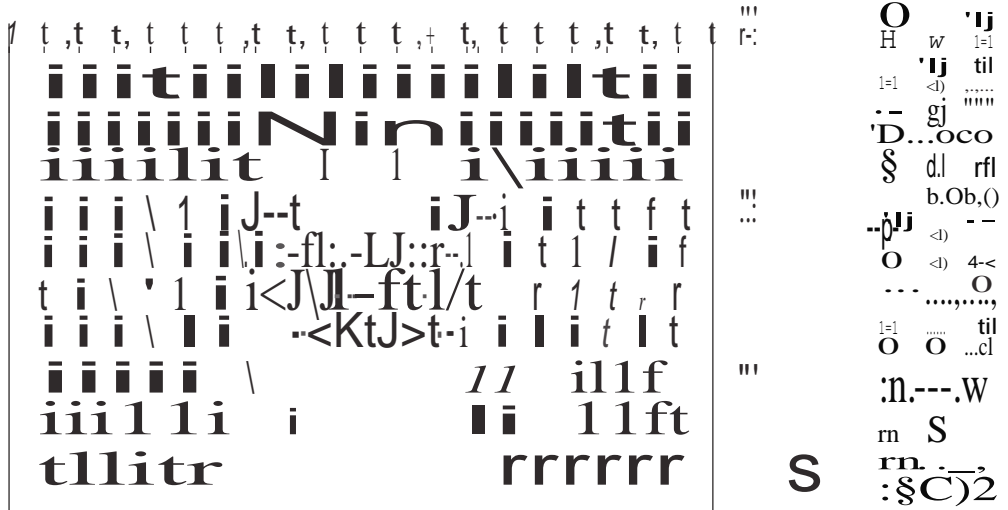

\section{fiifti \\ fifi 11 \\ trrrrrr \\ tiifiiiiiat \\ iijioisi i i \\ tfiiiftii \\ iffiiinffi \\ tetiiinitfi \\ tt \\ ftetiinteisil teti

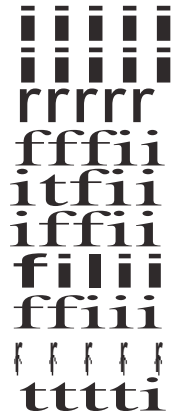

u.

$\mathrm{H} \quad \mathrm{c} \cdot 1.1$

til 8

1:1

$-1.0$

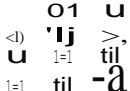

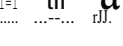

+u til

$\Rightarrow$ o....,

Cil i?: II

(ruYJ:) $£$.

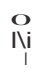

$\underset{\substack{a \\ i}}{\substack{a \\ i}}$

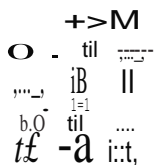


Table 2

Fluctuations' statistics; non-rotating low Froude number flow solutions

\begin{tabular}{ccccc}
\hline \hline$\Psi$ & $\max$ & $\min$ & average & std. dev. \\
$\mathrm{u}_{\mathrm{T}} ; \mathrm{u}_{\mathrm{R}}$ & $8.28 ; 8.71$ & $-6.42 ; 5.95$ & $-0.073 ; 0.052$ & $1.29 ; 0.804$ \\
$\mathrm{v}_{\mathrm{T}} ; \mathrm{v}_{\mathrm{R}}$ & $8.56 ; 8.74$ & $-8.63 ;-8.74$ & $<10^{-3} ;<10^{-8}$ & $0.756 ; 0.504$ \\
$\mathrm{w}_{\mathrm{T}} ; \mathrm{w}_{\mathrm{R}}$ & $6.22 ; 6.00$ & $-5.07 ;-5.19$ & $-0.032 ;-0.001$ & $0.619 ; 0.386$ \\
$\Theta_{\mathrm{T}} ; \Theta_{\mathrm{R}}$ & $3.13 ; 3.28$ & $-1.48 ;-1.60$ & $0.019 ;-0.005$ & $0.296 ; 0.218$ \\
$\mathrm{\eta}_{\mathrm{T}} ; \mathrm{\eta}_{\mathrm{R}}$ & $-1041 ;-1092$ & $493 ; 532$ & $-6.19 ; 1.67$ & $98.6 ; 72.6$ \\
\hline
\end{tabular}

Tables 2 and 3 quantify departures of the edge-based, $\Psi_{\mathrm{T}}$ (for Runs T), and the structured-grid, $\Psi_{\mathrm{R}}$ (for reference Runs $\mathrm{R}$ ), solutions for the non-rotating and rotating flow experiments, respectively. Statistics of fluctuations about the ambient state $\psi^{\prime}=\psi-\psi_{\mathrm{e}}$ - global extrema $\max \left(\psi^{\prime}\right)$ and $\min \left(\psi^{\prime}\right)$ together with domain averages $\left(\psi^{\prime}\right)$ and standard deviations $\left(\left(\psi^{\prime}-\left(\psi^{\prime}\right)\right)^{2}\right)^{1 / 2}$ - are shown for three velocity components and potential temperature using SI units of $\left[\mathrm{ms}^{-1}\right]$ and $[\mathrm{K}]$. Furthermore, for the reader convenience, we also convert statistics of $\Theta$ to vertical displacements of isentropes using crude approximation $\eta_{\mathrm{z}} \approx-\left(\Theta_{0} \mathrm{~N}^{2} / \mathrm{g}\right)^{-1} \Theta^{\prime} \approx-333 \Theta^{\prime}[\mathrm{m}]$.

Information contained in the tables substantiates our earlier assertions. Here, we draw the reader's attention to a few selected points. The overall agreement of the corresponding solutions for Runs $T$ and $R$ is apparent, and the biggest differences are in standard deviations of dependent variables fluctuations, showing more variability for the unstructured-mesh results. This should not be surprising as the refined mesh is unsymmetric, unlike the structured grid, and the flow responds to it accordingly. Noteworthy, even though the standard deviations of velocity fluctuations can differ between Runs $T$ and $R$ by tens of percent, they are still substantially smaller then the ambient flow $\left(u_{e}=5 \mathrm{~ms}^{-1}\right)$. The statistics of the isentrope displacements add to the earlier discussion about the dividing streamline (in the paragraph subsequent to eq.19). They show maximal upward displacement of about $\mathrm{F} \mathrm{h}_{\mathrm{o}}$ and a twice larger downward displacements consistent with sinking of the isentropes from above the hill summit on the lee slopes (hinted by the central panel of Fig. 7), familiar from the windstorms over tall 2D ridges [5]. Comparing the two tables shows that overall effects of planetary rotation are weak, albeit they tend to emphasize the south/north asymmetry of the solution seen in the extreme values' disparity of the spanwise flow.

We end the discussion of the results with a comment on the relative computational efficacy of the edge-based and the structured-grid model. Numerics wise, both models were configured similarly to enable a fair assessment of the economy of unstructured mesh calculations. In particular, all four experiments were performed with the same time step ensuring advective Courant numbers 
Table 3

Fluctuations' statistics; rotating low Froude number flow solutions

\begin{tabular}{ccccc}
\hline \hline & $\max$ & $\min$ & average & std. dev. \\
$\mathrm{u}_{\mathrm{T}} ; \mathrm{u}_{\mathrm{R}}$ & $9.63 ; 8.90$ & $-7.78 ; 6.94$ & $-0.069 ; 0.052$ & $1.23 ; 0.763$ \\
$\mathrm{v}_{\mathrm{T}} ; \mathrm{v}_{\mathrm{R}}$ & $7.00 ; 8.21$ & $-7.73 ;-8.02$ & $-0.029 ; 0.003$ & $0.866 ; 0.502$ \\
$\mathrm{w}_{\mathrm{T}} ; \mathrm{w}_{\mathrm{R}}$ & $6.08 ; 5.90$ & $-5.31 ;-5.01$ & $-0.031 ;-0.001$ & $0.613 ; 0.381$ \\
$\Theta_{\mathrm{T}} ; \Theta_{\mathrm{R}}$ & $3.38 ; 3.48$ & $-1.47 ;-1.46$ & $0.016 ;-0.005$ & $0.293 ; 0.215$ \\
$\eta_{\mathrm{T}} ; \mathrm{\eta}_{\mathrm{R}}$ & $-1126 ;-1159$ & $490 ; 486$ & $-5.33 ; 1.67$ & $97.6 ; 71.6$
\end{tabular}

slightly smaller than required by the stability limit, $C$;S 1 in terminology of structured grids [33], and all calculations converged to the same accuracy threshold $r_{u} \infty_{\infty}<10^{-5}$ in solution of the elliptic Poisson equations (section 3.2). Even though the unstructured refined mesh calculations required more work to achieve the same accuracy threshold, the overall comparison of the relative model economy is encouraging, although with a caveat. While the structured-grid model relies on massive parallelizm with a proven record of high performance [23,21], the newly developed edge-based model still runs in a serial mode, thus making a direct comparison of the two codes unfair. For illustration, each structured-grid simulations of the low Froude number flows discussed in this section takes about 40s of the wallclock time on the 88 cores of IBM Power 575 supercomputer, whereas corresponding calculations with the edge-based code take somewhat less than one hour (exact wallclock time was not measured) of a single processor Dell Precision 690n workstation. Nonetheless, when accounting for the disparity of the computer power exploited by both codes, the overall computational work appears roughly the same. This shows the competitiveness of the unstructured mesh model given an efficient parallelization scheme - a work that is currently pursued.

\section{REMARKS}

In this paper a class of new three-dimensional edge-based non-hydrostatic atmospheric models is presented. The models extend the applicability of NFTMPDATA solvers to fully unstructured meshes capable of modeling complex geometrical configurations. In addition to the three dimensional generalization, this work extends our earlier edge-based unstructured mesh model developments by introducing Coriolis effects free of hydrostatic simplifications, and surface boundary layer forcings that improve the correspondence of the explicit and implicit large eddy simulations discussed earlier in the literature.

The developed solvers show quantitative agreement with laboratory and field experiments and computations obtained from the proven structured-grid NFT- 
MPDATA model. The simulated canonical flow cases of evolutionary convective Planetary Boundary Layer and strongly stratified orographic flows are well studied and challenging. Quality solutions of these problems are documented for unstructured meshes, to our best knowledge, for the first time ever. A thorough analysis of the results confirms that the unstructured mesh solvers have the ability to match the accuracy of structured-grid models in simulation of intricate turbulent atmospheric flows, and indicates the potential for a substantial reduction in the number of mesh points without compromising the accuracy of solution.

The results obtained confirm that unstructured-mesh models provide viable computational tools for simulating small to mesoscale thermal and mechanical effects comprising highly nonlinear nonhydrostatic atmospheric dynamics. The flexibility of the mesh-refinement offered by the edge-based codes opens new opportunities for research, parameterization, and prognosis of diverse natural phenomena, many of which still elude complete understanding.

Acknowledgments: The authors are gratful to Prof. Rupert Klein for a thor- ough review and insightful comments. The authors acknowledge support by the Newton Institute visitor fellowships, DOE award \#DE-SC0006748, NERC/G004358 award, the NSF grant OCI-0904599, and by funding received from the European Research Council under the European Union's Seventh Framework Programme (FP7/2012/ERC Grant agreement no. 320375). The National Center for Atmospheric Research is sponsored by the National Science Foundation.

\section{References}

[1] V. Balaji, J-L. Redelsperger, G.P. Klaassen, Mechanisms for the mesoscale organization of tropical cloud clusters in GATE Phase-III. 1. Shallow Cloud Bands, J. Atmos. Sci., 50 (1993) 3571-3589.

[2] J. Behrens, S. Reich, M. Laüter, B. Wingate, D. Williamson, The 2010 Workshop on the Solution of PDEs on the Sphere; http://www.awipotsdam.de/pde2010/pdes2010_program.pdf

[3] T.L. Clark, R.D. Farley, Severe downslope windstorm calculations in two and three spatial dimensions using anelastic interactive grid nesting: a possible mechanism for gustiness, J. Atmos. Sci., 41 (1984) 329-350.

[4] J.W. Deardorff, G.E. Willis, Further results from a laboratory model of the convective planetary boundary layer, Boundary-Layer Met. 32 (1985) 205-236.

[5] J.D. Doyle, S. Gabersek, QF. Jiang, L. Bernardet, J.M. Brown, A. Dörnbrack, E. Filaus, V. Grubišić, D.J. Kirshbaum, O. Knoth, S. Koch, J. Schmidli, I. Stiperski, S. Vosper, and SY. Zhong, Intercomparison of T-REX mountain-wave 
simulations and implications for mesoscale predictability, Month. Weather Rev. 139 (2011) 2811-2831.

[6] D.R. Durran, Improving the anelastic approximation, J. Atmos. Sci. 46 (1989) 1453-1461.

[7] C.C. Epifanio, R. Rotunno, The dynamics of orographic wake formation in flows with upstream blocking, J. Atmos. Sci. 62 (2005) 3127-3150.

[8] M. Ghizaru, P. Charbonneau, P.K. Smolarkiewicz, Magnetic Cycles in Global Large-eddy Simulations of Solar Convection, Astrophys. J. Lett. 715 (2010) L133L137

[9] W.W Grabowski, P.K. Smolarkiewicz, A multiscale anelastic model for meteorological research, Month. Weather Rev. 130 (2002), 939-956.

[10] T. Hauf T.L. Clark, 3-dimensional numerical experiments on convectively forced internal gravity waves, Q.J. Roy. Meteor. Soc. 115 (1989), 309-333.

[11] C.R. Hunt, W.H. Snyder, Experiments on stably and neutrally stratified flow over a model three-dimensional hill, J. Fluid Mech. 96 (1980) 671-704.

[12] J.D. Hyman, P.K. Smolarkiewicz, C.L. Winter, Heterogeneities of flow in stochastically generated porous media, Phys. Rev. E 86 (2012) 056701.

[13] R. Klein, U. Achatz, D. Bresch, O.M. Knio, P.K. Smolarkiewicz (2010), Regime of validity of soundproof atmospheric flow models, J. Atmos. Sci. 67 (2010) 32263237.

[14] C. Kühnlein, P.K. Smolarkiewicz, A. Dörnbrack, Modelling atmospheric flows with adaptive moving meshes, J. Comput. Phys. 231 (2012) 2741-2763.

[15] D.H. Lenschow, J.C. Wyngaard, W.T. Pennel, Mean-field and second-moment budgets in a baroclinic, convective boundary layer, J. Atmos. Sci. 37 (1980) 1313-1326.

[16] F.B. Lipps, R.S. Hemler, A scale analysis of deep moist convection and some related numerical calculations, J. Atmos. Sci. 39 (1982) 2192-2210.

[17] F.B. Lipps, On the anelastic approximation for deep convection, J. Atmos. Sci. 47 (1990) 1794-1798.

[18] L.G. Margolin, P.K. Smolarkiewicz, Z. Sorbjan, Large-eddy simulations of convective boundary layers using nonoscillatory differencing, Physica D 133 (1999) 390-397.

[19] Predicting weather, climate and extreme events, J. Comput. Phys. 227, Spec. Issue 7, (2008) 3429-3730, Eds. M.J. Miller, P.K. Smolarkiewicz.

[20] Z.P. Piotrowski, P.K. Smolarkiewicz, S.P. Malinowski, A.A. Wyszogrodzki, On numerical realizability of thermal convection, J. Comput. Phys. 228 (2009) 62686290. 
[21] Z.P. Piotrowski, A.A. Wyszogrodzki, P.K. Smolarkiewicz, Towards petascale simulations of atmospheric circulations with soundproof models, Acta Geophysica 59 (2011) 1294-1311.

[22] J.M. Prusa, P.K. Smolarkiewicz, An all-scale anelastic model for geophysical flows: dynamic grid deformation, J. Comput. Phys. 190 (2003) 601-622.

[23] J.M. Prusa, P.K. Smolarkiewicz, A.A Wyszogrodzki, EULAG, a computational model for multiscale flows, Comput. Fluids 37 (2008) 1193-1207

[24] H. Schmidt, U. Schumann, Coherent structure of the convective boundary layer derived from large-eddy simulation, J. Fluid Mech. 200 (1989) 511-562.

[25] W.C. Skamarock, J.B. Klemp, M.G. Duda, L.D. Fowler, S.-H. Park, A multiscale nonhydrostatic atmospheric model using centroidal Voronoi tesselations and C-grid staggering, Month. Weather Rev. 140 (2012) 3090-3105.

[26] R.B. Smith, Linear theory of hydrostatic flow over an isolated mountain in isosteric coordinates, J. Atmos. Sci. 45 (1988) 3887-3896.

[27] P.K. Smolarkiewicz, R. Rotunno, Low Froude number flow past threedimensional obstacles. Part I: Baroclinically generated lee vortices, J. Atmos. Sci. 46 (1989) 1154-1164.

[28] P.K. Smolarkiewicz, L.G. Margolin, On forward-in-time differencing for fluids: Extension to a curvilinear framework, Month. Weather Rev. 121 (1993) 18471859.

[29] P.K. Smolarkiewicz, L.G. Margolin, MPDATA: A finite-difference solver for geophysical flows, J. Comput. Phys. 140 (1998) 459-480.

[30] P.K. Smolarkiewicz, L.G. Margolin, A.A Wyszogrodzki, A class of nonhydrostatic global models, J. Atmos. Sci. 58 (2001) 349-364.

[31] P.K. Smolarkiewicz, J.M. Prusa, Towards mesh adaptivity for geophysical turbulence: continuous mapping approach, Int. J. Numer. Meth. Fluids 47 (2005) 789-801.

[32] P.K. Smolarkiewicz, J. Szmelter, Multidimensional positive definite advection transport algorithm (MPDATA) : an edge-based unstructured-data formulation, Int. J. Numer. Meth. Fluids 47 (2005) 1293-1299.

[33] P.K. Smolarkiewicz, J. Szmelter, MPDATA: An Edge-Based Unstructured-Grid Formulation, J. Comput. Phys. 206 (2005) 624-649.

[34] P.K. Smolarkiewicz, Multidimensional positive definite advection transport algorithm: an overview, Int. J. Numer. Meth. Fluids 50 (2006) 1123-1144.

[35] P.K. Smolarkiewicz, R. Sharman, J. Weil, S.G. Perry, D. Heist, G. Bowker, Building resolving large-eddy simulations and comparison with wind tunnel experiments, J. Comput. Phys. 227 (2007) 633-653.

[36] P.K. Smolarkiewicz, J. Szmelter, Iterated upwind schemes for gas dynamics, J. Comput. Phys. 228 (2009) 33-54. 
[37] P.K. Smolarkiewicz, Modeling atmospheric circulations with soundproof equations. Proceedings of the ECMWF Workshop on Nonhydrostatic Modelling, 8-10 November, 2010, Reading, UK (2011) 1-15.

[38] P.K. Smolarkiewicz, J. Szmelter, A nonhydrostatic unstructured-mesh soundproof model for simulation of internal gravity waves, Acta Geophysica 59 (2011) 1109-1134.

[39] G. Strang, On the construction and comparison of difference schemes, SIAM J. Numer. Anal. 5 (1968) 506-517.

[40] J. Szmelter, P.K. Smolarkiewicz, MPDATA error estimator for mesh adaptivity, Int. J. Numer. Meth. Fluids 50 (2006) 1269-1293.

[41] J. Szmelter, P.K. Smolarkiewicz, An edge-based unstructured mesh discretisation in geospherical framework, J. Comput. Phys. 229 (2010) 4980-4995.

[42] J. Szmelter, P.K. Smolarkiewicz, An edge-based unstructured mesh framework for atmospheric flows, Comput. Fluids. 46 (2011) 455-460.

[43] M.L. Waite, P.K. Smolarkiewicz, Instability and breakdown of a vertical vortex pair in a strongly stratified fluid, J. Fluid Mech. 606 (2008) 239-273.

[44] N.P. Wedi, P.K. Smolarkiewicz, Extending Gal-Chen and Somerville terrainfollowing coordinate transformation on time dependent curvilinear boundaries, J. Comput. Phys. 193 (2004) 1-20.

[45] D.L. Williamson, The evolution of dynamical cores for global atmospheric models, J. Meteor. Soc. Japan 85B (2008) 241-268. 